\title{
Polaronic exciton in a parabolic quantum dot
}

\author{
R. T. Senger* and K. K. Bajaj \\ Department of Physics, Emory University, Atlanta, Georgia 30322, USA
}

Received 17 July 2002, revised 9 October 2002, accepted 10 October 2002 Published online 13 February 2003

PACS 71.35.-y, 71.38.-k, 73.21.La

\begin{abstract}
A calculation of the variations of the energy and oscillator strength of the ground state of an exciton in an ionic quantum dot as a function of the dot size, using a parabolic confining potential and a variational approach, is presented. The strong exciton-phonon interaction is taken into account by using an effective potential between the electron and the hole as derived by Pollmann and Büttner. The values of the exciton binding energies calculated using this potential are compared with those obtained using statically screened Coulomb potential and are found to be considerably larger for all values of the quantum dot size. In small quantum dots, the electron-hole correlation is reduced as the single particle energies scale at a much faster rate than the interaction energy. This is manifested in the behavior of oscillator strength, which becomes less dependent on the form of the effective interaction as the quantum dot size is reduced.
\end{abstract}

\section{Introduction}

With the advances in modern fabrication techniques, it has become possible to grow low-dimensional systems such as quantum wires and quantum dots with well-controlled dimensions and compositions. These structures, where quantum mechanical effects are strongly manifested, have become the subject of intensive experimental and theoretical investigations during the past decade [1]. The inherent fundamental physics and the potential use in designing more efficient microelectronic devices are among the two motivations of strong ongoing interest in these highly confined systems. In particular, quantum dots are used as the key structure in some important applications, such as the recent realization of ideal singlephoton source, which uses the anharmonicity of multi-exciton transitions in a single quantum dot [2]. In fact, the optical properties of quantum dots have been known to be promising for some time. For instance, quantum dot-based laser devices are expected to have superior performance [3].

In recent years there has been a great surge of interest in studying the electronic and optical properties of quantum dots based on wide bandgap semiconductors for their potential applications in optoelectronic devices in the blue and ultraviolet regions of the spectrum. Quantum dots based on GaN [4], $\mathrm{ZnSe}$ [5], $\mathrm{ZnO}$ [6], $\mathrm{MgO}$ [7], $\mathrm{CuCl}[8]$ and $\mathrm{CdS}$ [9] have been fabricated and their properties are studied in considerable detail. It has been suggested that the lasing mechanism in these systems is excitonic in nature and therefore can lead to lower values of the threshold current densities. Consequently, a proper understanding of the exciton related phenomena in these confined structures is of considerable importance. Of particular interest are the recent studies of polaronic effects on quasi-zero-dimensionally confined excitons [10-16]. Interestingly, the conclusions on the size dependence of the exciton-LO-phonon interaction in a quantum dot system are not uniform and even contradictory. Some groups point out that Fröhlich type exciton-LO-phonon interaction should vanish in small nanocrystals. However, in another study, using a donor-like exciton model and adiabatic approximation it has been concluded that exciton-

\footnotetext{
* Corresponding author: e-mail: senger@physics.emory.edu
} 
LO-phonon coupling strength is independent of dot radius. This was contradicted in another work, which claims that polaronic effects in a donor like exciton should increase with decreasing dot size (for further discussion see Ref. [15] and the references cited therein). In view of these divergent conclusions, the problem of exciton-phonon interaction in a quantum dot deserves further attention.

In polar semiconductors the excitonic properties are significantly altered due to the influence of the exciton-optical-phonon interaction. For instance, exciton binding energy is enhanced, depending on the strength of this interaction. A proper description of the nature of this coupling among electrons, holes, and phonons should not be oversimplified. This effect must be properly described by an effective electron-hole interaction and not by simply assuming that the electron and the hole band masses are renormalized to their respective polaron masses. In fact, the polaron-like interaction between electrons and holes depends on their mutual distance in such a way that when this is much larger than their polaron radii, the electron and the hole interact like polarons through the statically screened Coulomb potential. In the opposite limit, however, when their distance is less than their polaron radii, the two oppositely polarized virtual phonon clouds around each particle overlap and partially cancel out their renormalization effects, so that the electron-hole interaction approaches the dynamically screened Coulomb interaction. Such a description of the effective electron-hole interaction, encompassing the above mentioned limiting cases has already been given through some effective potentials [17-21].

In this paper, we present a variational calculation of the exciton binding energy and oscillator strength in quantum dots made up of polar materials. The exciton-optical phonon interaction is accounted for by means of the effective potential derived by Pollmann and Büttner (PB) $[18,19]$. As a model of quasizero-dimensional confinement a spherically symmetric parabolic potential is used. We show that the quantum dot type confinement has drastic effects on both the ground state energy and the oscillator strength of the exciton. The description of an excitonic state with the screened Coulomb interaction potential is known to have a limited validity, leading to reasonable quantitative results only for weakly polar materials such as GaAs. With the use of an appropriate effective interaction potential the exciton in a polar quantum dot will be shown to be much more compactly bound, with higher binding energies, and in particular with very pronounced oscillator strengths.

In the next section we provide the theoretical framework of our calculation. The results for three selective polar materials $(\mathrm{GaN}, \mathrm{ZnO}$, and $\mathrm{CuCl})$ and a brief discussion of $\mathrm{PB}$ effective potential together with confinement effects will form the last section.

\section{Theory}

The Hamiltonian of an interacting electron-hole pair, perfectly confined in a parabolic quantum dot, and in interaction with the bulk LO-phonons is given by

$$
H=\sum_{i=e, h}\left(\frac{p_{i}^{2}}{2 m_{i}}+\frac{1}{2} m_{i} \Omega^{2} r_{i}^{2}\right)-\frac{e^{2}}{\varepsilon_{\infty}\left|\boldsymbol{r}_{e}-\boldsymbol{r}_{h}\right|}+H_{\mathrm{ph}}+H_{\mathrm{exc}-\mathrm{ph}},
$$

where, the subscript $i=e$ and $i=h$ denotes an electron and a hole, respectively, $m_{i}$ are the isotropic effective masses, $\boldsymbol{r}_{i}$ are the position, and $\boldsymbol{p}_{i}$ are the momentum operators. The Coulomb interaction potential is screened with high frequency dielectric constant $\varepsilon_{\infty}$. The terms $H_{\mathrm{ph}}$ and $H_{\mathrm{exc}-\mathrm{ph}}$ describe the bulk longitudinal optical (LO) phonons, and the well-known Fröhlich-type interaction of the electron and the hole with the dispersionless phonons of frequency $\omega_{L O}$, respectively. In the present study, we consider the exciton to be interacting with the bulk phonons only, rather than explicitly dealing with confined, surface or interface phonon modes. Such an approximation is motivated by the fact that in quantum wells the interaction of an exciton with all three phonon modes (confined, interface, half-space) can effectively be reduced, with a good degree of accuracy, to a bulk-phonon-exciton interaction, as it has been shown by several groups [22-26].

Transformation to center-of-mass and relative coordinates, $\boldsymbol{R}=\left(m_{e} \boldsymbol{r}_{e}+m_{h} \boldsymbol{r}_{h}\right) / M$ and $\boldsymbol{r}=\boldsymbol{r}_{e}-\boldsymbol{r}_{h}$, respectively, separates the kinetic and potential energy terms of the Hamiltonian. The part corresponding to the 
center-of-mass motion has the form of a simple harmonic oscillator with the total mass $M=m_{e}+m_{h}$, which contributes subband energy of $3 / 2 \hbar \Omega$ to the total ground state energy. We express the remaining part, which turns out to be the effective Hamiltonian of the system, in terms of the relative coordinates only:

$$
H_{\mathrm{eff}}=-\frac{\hbar^{2}}{2 m_{\mu}} \nabla_{r}^{2}+\frac{1}{2} m_{\mu} \Omega^{2} r^{2}+V_{\mathrm{eff}}(r)+E_{\mathrm{self}}
$$

where $m_{\mu}=m_{e} m_{h} / M$ is the exciton reduced mass and the last two terms $V_{\text {eff }}(r)$ and $E_{\text {self }}$ describe the effective electron-hole interaction, and the polaronic self energy, respectively. The effective Hamiltonian given in Eq. (2) without the confinement potential is derived by Pollmann and Büttner [18, 19] and successfully explains the measured values of the exciton binding energies in bulk ionic semiconductors. The polaronic terms are given in the form

$$
\begin{aligned}
& V_{\text {eff }}(r)=-\frac{e^{2}}{\varepsilon_{0} r}-\frac{e^{2}}{\varepsilon^{*} r}\left[\frac{C^{4}}{B^{4}}-\frac{m_{e} h_{e}}{\Delta m} \mathrm{e}^{-r A_{e} / R_{e}}+\frac{m_{h} h_{h}}{\Delta m} \mathrm{e}^{-r A_{h} / R_{h}}-\left(h_{\mu}+\frac{C^{3} r}{2 B^{3} a_{\text {ex }}}\right) \mathrm{e}^{-r B / R_{\mu}}\right], \\
& E_{\text {self }}=-\left(\alpha_{e} g_{e}+\alpha_{h} g_{h}-\alpha_{\mu} g_{\mu}\right) \hbar \omega_{\mathrm{LO}}
\end{aligned}
$$

where $\varepsilon^{*}=\left(1 / \varepsilon_{\infty}-1 / \varepsilon_{0}\right)^{-1}, \varepsilon_{0}$ is the static dielectric constant, $\Delta m=m_{h}-m_{e}$, and

$$
\alpha_{i}=\frac{e^{2}}{2 \varepsilon^{*} R_{i} \hbar \omega_{\mathrm{LO}}}, \quad R_{i}=\sqrt{\frac{\hbar}{2 m_{i} \omega_{\mathrm{LO}}}}, \quad(i=e, h, \mu)
$$

are the dimensionless phonon-coupling constants, and the characteristic polaron radii for the electron, the hole, and the reduced mass $m_{\mu}$, respectively. The remaining coefficients have the following explicit forms:

$$
\begin{aligned}
& A_{i}^{2}=1+R_{i}^{2} / a_{\mathrm{ex}}^{2}, \quad(i=e, h) \\
& B^{2}=1+C^{2}, \quad C^{2}=R_{\mu}^{2} / a_{\mathrm{ex}}^{2} ; \\
& h_{i}=1+\left(m_{j} R_{i} / m_{i} a_{\mathrm{ex}}\right)^{2}, \quad(j \neq i=e, h) ; \\
& h_{\mu}=-\left(m_{e} / m_{h}+m_{h} / m_{e}\right) C^{2}+C^{4} / B^{4} ; \\
& g_{i}=A_{i}\left(1-\frac{1}{2} R_{i}^{2} / a_{\mathrm{ex}}^{2}\right)^{2}, \quad(i=e, h) ; \\
& g_{\mu}=C^{2}\left(4+\frac{1}{2} C^{2}+C^{2} / B^{2}-2 A_{e} d_{e}-2 A_{h} d_{h}\right) / B ; \\
& d_{i}=\left(1+A_{i} B \sqrt{m_{j} / M}\right) /\left(A_{i}+B \sqrt{m_{j} / M}\right), \quad(j \neq i=e, h) .
\end{aligned}
$$

In the above, all the terms have dependence on a quantity, exciton radius $a_{\mathrm{ex}}$, which is to be determined variationally.

Originally the Pollmann-Büttner effective potential between the electron and the hole was derived for a bulk semiconductor using a one parameter hydrogenic trial wavefunction, $\Psi_{0}(r)=N \exp \left(-\lambda r / a_{0}\right)$, where $a_{0}=\hbar^{2} \varepsilon_{0} / m_{\mu} e^{2}$ is the exciton Bohr radius. Consequently, the value of the effective exciton radius used in the effective Hamiltonian has a bulk limit given by $a_{\mathrm{ex}}=\left\langle\Psi_{0}|1 / r| \Psi_{0}\right\rangle^{-1}=a_{0} / \lambda$. In the case of confinement, however, the variational trial wave function we use contains a Gaussian envelope function, which is compatible with the nature of the dot-potential geometry:

$$
\Psi(r)=N \exp \left(-\lambda r / a_{0}\right) \exp \left(-v r^{2} / R_{d}^{2}\right) .
$$


In the above, $N$ is the normalization constant, $\lambda$ and $v$ are the two variational parameters, and $R_{d}=\sqrt{\hbar / 2 m_{\mu} \Omega}$ is a characteristic length of the confinement which we will define as the effective radius of the quantum dot. With the number of variational parameters increased from one to two, the exciton radius to be used in the effective Hamiltonian should now depend on both of them: $a_{\mathrm{ex}}=\langle\Psi|1 / r| \Psi\rangle^{-1}$.

We should also note that in this treatment of the effective electron-hole interaction, the renormalization of the electron and hole masses is not needed as shown by Pollmann and Büttner in their work [19]. We assume that the above form of the effective potential, which is derived for bulk, is not significantly modified in the presence of the external confinement. Such an approximation has been used successfully in the case of a polaronic exciton in a magnetic field [27].

To calculate the ground state energy, the expectation value of the effective Hamiltonian, Eq. (2) is minimized with respect to the variational parameters $\lambda$ and $v$. We define the quantity $E_{B}$ as the exciton binding energy, which is given with respect to the subband energy of the parabolic potential, and the self energies of the electron and the hole polarons,

$$
E_{B}=\frac{3}{2} \hbar \Omega-\left(\alpha_{e}+\alpha_{h}\right) \hbar \omega_{\mathrm{LO}}-\min _{\lambda, v}\left\langle\Psi\left|H_{\mathrm{eff}}\right| \Psi\right\rangle .
$$

The expectation value of the effective Hamiltonian is analytical, but the final expression is rather lengthy and will not be presented here.

We feel that the binding energy of an exciton in a quantum dot needs a clarified definition. In general, the term "binding energy" refers to the amount of energy required to break the bound state of the e-h system and to put the constituent particles in their one-particle states. Such a transition is possible in bulk, in a quantum-well, and even in a quantum-wire, where the electron-hole pair can be spatially separated in at least one free dimension. However, in a quantum-dot type confinement, where the exciton is considered to be perfectly confined inside the dot, the electron and the hole cannot be found in uncorrelated one-particle states. Therefore, the quantity defined in Eq. (8) actually corresponds to the Coulomb correlation energy of the exciton. In the following, the term "binding energy" should be interpreted within this context.

For comparison purposes we also consider the simpler model of polaronic exciton, in which the Coulomb interaction between the electron and hole is statically screened by $\varepsilon_{0}$, and the self-energy term is taken as the sum of individual polaron self-energy shifts of the electron and the hole. Neglecting the effects due to the overlapping of the polarization fields of the particles entirely, this model is valid in the shallow exciton limit only. We will denote the exciton binding energy calculated with the static screened interaction model by $E_{B}^{(s)}$, and similarly the other corresponding quantities will be referred to with the superscript $(s)$.

\section{Results and discussion}

We have calculated the variation of the exciton binding energy as a function of the radius of the dot in $\mathrm{GaN}, \mathrm{ZnO}$, and $\mathrm{CuCl}$. The values of the various physical parameters used in our calculations are given in Table 1. In Fig. 1 we display our results in these three systems. The solid curves are obtained using PB potential and the dashed curves are obtained using a static screened Coulomb potential. First we find that the value of the exciton binding energy increases as the dot size is reduced in both cases, as expected. And second, the value of the exciton binding energy in a given material obtained using PB potential is always larger than its value calculated using static screened potential.

In Fig. 2 we plot the difference between $E_{B}$ and $E_{B}^{(s)}$ as a function of $R_{d}$. We find that as the value of $R_{d}$ is reduced this difference stays about the same until $R_{d}$ becomes comparable to the effective exciton radius. This difference then increases rapidly as the value of $R_{d}$ is reduced further. It is clear that the deviations from the static screening case, namely, the importance of the polaronic effects can be very 

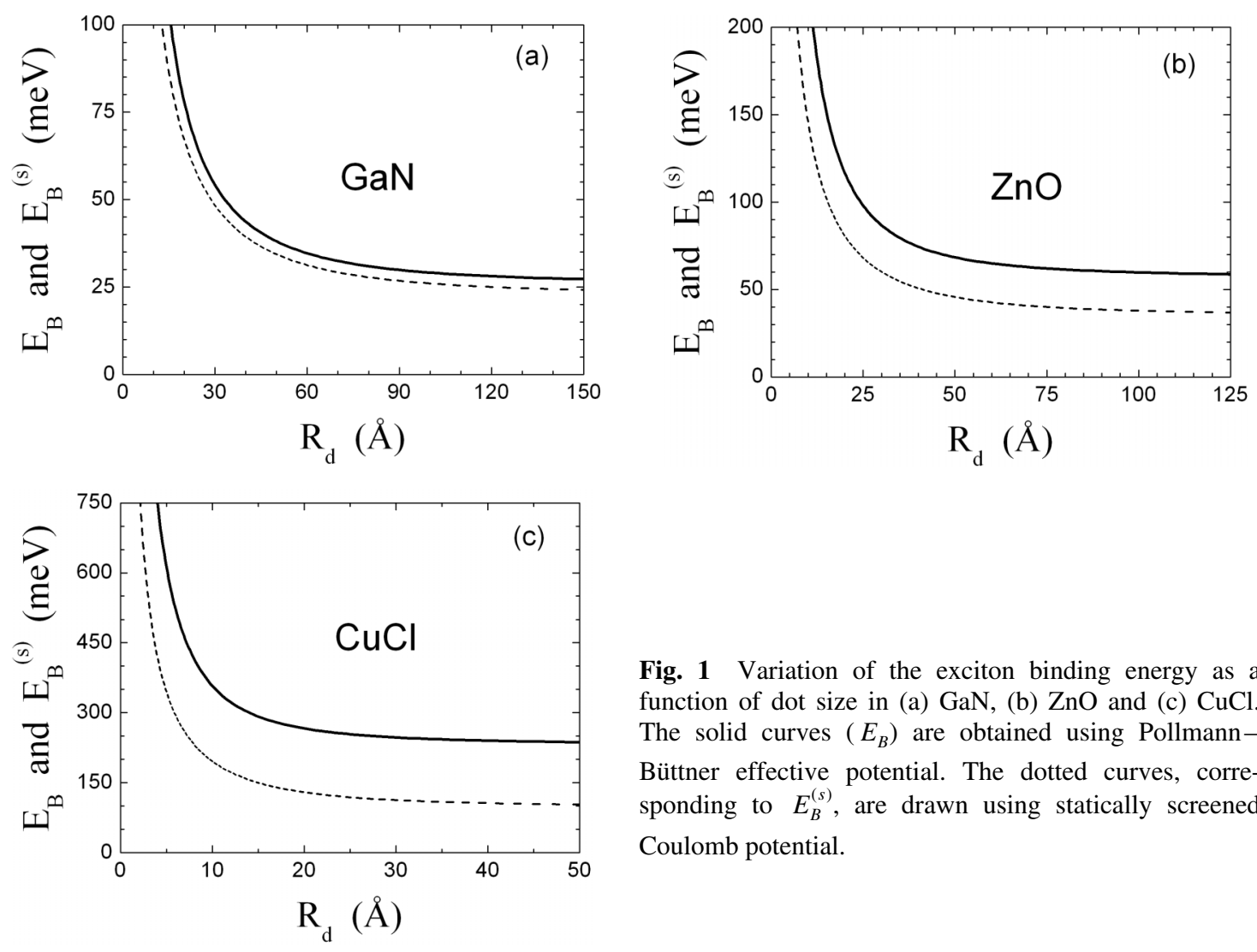

Fig. 1 Variation of the exciton binding energy as a function of dot size in (a) $\mathrm{GaN}$, (b) $\mathrm{ZnO}$ and (c) $\mathrm{CuCl}$. The solid curves $\left(E_{B}\right)$ are obtained using PollmannBüttner effective potential. The dotted curves, corresponding to $E_{B}^{(s)}$, are drawn using statically screened Coulomb potential.

striking for highly polar materials such as $\mathrm{ZnO}$ and $\mathrm{CuCl}$. For the case of $\mathrm{CuCl}$, for example, the binding energy calculated with PB potential is much larger and the effective exciton size is much smaller than their corresponding values obtained with the statically screened Coulomb potential. The correction brought about by the PB potential is not significant for weakly polar materials such as GaAs, but it be comes quite striking as the ionic character of the crystal increases. However even for GaAs, in very small quantum dots $\left(R_{d} \sim 0.1 a_{0}\right)$, the predicted enhancement in exciton binding energy using PB potential is about $10 \%$.

As shown in Fig. 1, even in the bulk limit, $R_{d} \gg a_{0}$, the enhancement in the exciton binding energy brought about by using the PB potential is significant. With the inclusion of PB effective potential, the value of the exciton binding energy in $\mathrm{GaN}$ increases by $13.5 \%$, in $\mathrm{ZnO}$ by $63 \%$, and in $\mathrm{CuCl}$ by as much as $139 \%$. For a qualitative understanding of such an enhancement in the values of exciton binding energy, it is instructive to consider the form and the limiting cases of the PB effective Hamiltonian.

Table 1 Values of the various physical parameters used in the calculation. The electron and the hole masses are expressed in terms of the free electron mass. The LO-phonon energies are given in meV. Symbols are defined in the text.

\begin{tabular}{lllllllllll}
\hline Material & $\begin{array}{l}m_{e} \\
\left(m_{0}\right)\end{array}$ & $\begin{array}{l}m_{h} \\
\left(m_{0}\right)\end{array}$ & $\varepsilon_{0}$ & $\varepsilon_{\infty}$ & $\begin{array}{l}\hbar \omega_{\mathrm{LO}} \\
(\mathrm{meV})\end{array}$ & $\alpha_{e}$ & $\alpha_{h}$ & $\begin{array}{l}R_{e} \\
(\AA)\end{array}$ & $\begin{array}{l}R_{h} \\
(\AA)\end{array}$ & $\begin{array}{l}a_{0} \\
(\AA)\end{array}$ \\
\hline $\mathrm{GaN}^{\mathrm{a}}$ & 0.2 & 0.8 & 9.8 & 5.4 & 92.0 & 0.45 & 0.90 & 14.4 & 7.2 & 32.4 \\
$\mathrm{ZnO}^{\mathrm{b}}$ & 0.28 & 0.59 & 8.6 & 4.0 & 72.0 & 0.97 & 1.41 & 13.7 & 9.5 & 24.0 \\
$\mathrm{CuCl}^{\mathrm{c}}$ & 0.44 & 3.6 & 7.4 & 3.7 & 27.2 & 2.01 & 5.73 & 17.8 & 6.2 & 9.99 \\
\hline
\end{tabular}

${ }^{\mathrm{a}}$ Ref. 31, 32; ${ }^{\mathrm{b}}$ Ref. 18, 21; ${ }^{\mathrm{c}}$ Ref. 21, 33 


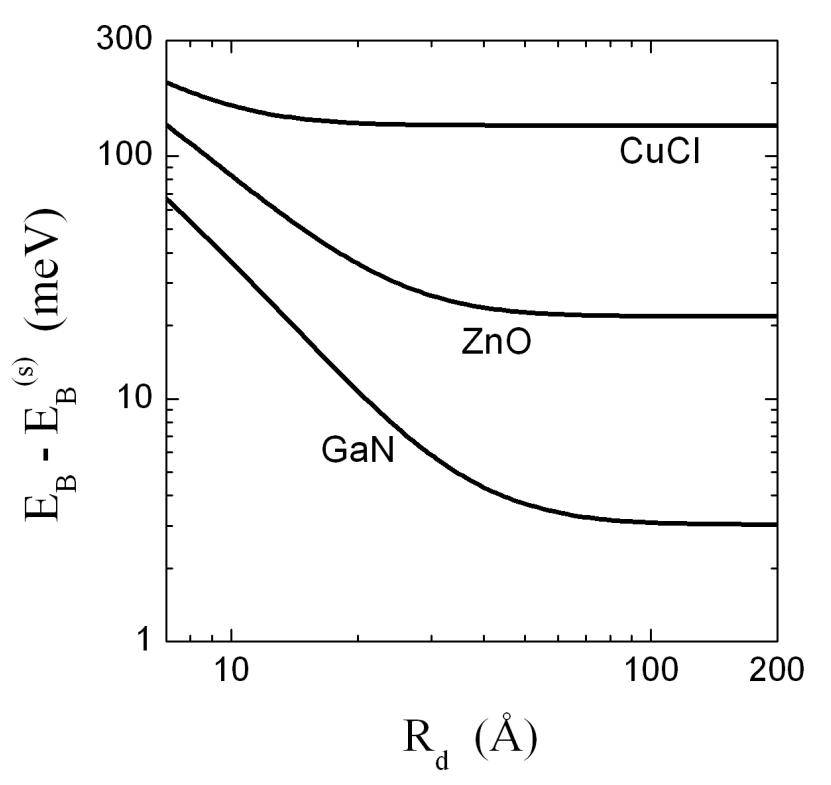

Fig. 2 Variation of the difference between $E_{B}$ and $E_{B}^{(s)}$ as a function of quantum dot size in $\mathrm{GaN}, \mathrm{ZnO}$ and $\mathrm{CuCl}$.

The first term in Eq. (3) corresponds to a statically screened Coulomb interaction. As compared to the original form of the Hamiltonian (1), the elimination of phonon coordinates to obtain an effective electron-hole interaction transforms the Coulomb interaction from a dynamically screened to a statically screened one, in the leading order. However the remaining terms in Eq. (3) as well as the self-energy expression, Eq. (4), are essential to describe the detailed and non-trivial interaction of the two charges with opposite polarization fields around them. The crucial parameter that determines the form of the effective interaction is the ratio of the exciton radius $a_{\text {ex }}$ to the polaron radii $R_{i}$. It is instructive to check the limiting cases of this ratio in an ionic material. In the limits of weak and strong binding it is easy to show that

$$
\lim _{a_{\mathrm{ex}} / R_{i} \rightarrow \infty} E_{\mathrm{self}}=-\left(\alpha_{e}+\alpha_{h}\right) \hbar \omega_{\mathrm{LO}}, \quad \text { and } \quad \lim _{a_{\mathrm{ex}} / R_{i} \rightarrow 0} E_{\mathrm{self}}=0 .
$$

For large exciton radii the self-energy approaches the free polaron values. For very small exciton radii the self-energy of the exciton vanishes because the polarization clouds of the electron and the hole cancel each other. Similarly,

$$
\lim _{a_{\mathrm{ex}} / R_{i} \rightarrow \infty} V_{\text {eff }}(r)=-\frac{e^{2}}{\varepsilon_{0} r}-\frac{e^{2}}{\varepsilon^{*} r \Delta m}\left(m_{h} \mathrm{e}^{-r / R_{h}}-m_{e} \mathrm{e}^{-r / R_{e}}\right) \quad \text { and } \quad \lim _{a_{\mathrm{ex}} / R_{i} \rightarrow 0} V_{\text {eff }}(r)=-\frac{e^{2}}{\varepsilon_{\infty} r} .
$$

With these reasonable limits, PB Hamiltonian gives a successful description of polaronic excitons [19, 27]. In the present study the main effect of the confining potential is to shrink the charge densities of both the electron and the hole, decreasing the relative distance between them; which in turn makes the effective electron-hole interaction potential to be less screened through the PB term. Therefore, the high degree of confinement leads to vanishing of polaronic effects in the exciton state. The same conclusion has been reached by another group in their recent work [15] on size dependence of polaronic effects on an exciton in a quantum dot.

Another quantity of interest is the oscillator strength, which is sensitively dependant on the confinement of the electron and hole wavefunctions and the overlap between them. Using the envelope-function approximation, the oscillator strength for the exciton ground state is given by [28-30]

$$
f_{\text {ex }}=\frac{2 P^{2}}{m_{0} \Delta E}\left|\int \psi_{\text {ex }}\left(r_{e}, r_{e}\right) \mathrm{d} r_{e}\right|^{2}
$$


where $P$ describes the intracell matrix-elements, $m_{0}$ is the bare electron mass, $\Delta E=E_{\text {ex }}-E_{0}, E_{\text {ex }}$ and $E_{0}$ are the energies of the states with and without the exciton, respectively. Since we are interested in comparing the effect of using PB potential on the oscillator strength with that of using the screened Coulomb potential, we calculate the ratio of the two oscillator strengths. This ratio can be expressed in terms of the energies and the wavefunctions corresponding to the relative coordinates only,

$$
\frac{f_{\mathrm{ex}}}{f_{\mathrm{ex}}^{(s)}}=\frac{\Delta E^{(s)}}{\Delta E} \frac{|\Psi(0)|^{2}}{\left|\Psi^{(s)}(0)\right|^{2}} .
$$

In Fig. 3 we plot the variation of this ratio of two oscillator strengths as a function of the dot size. We see a trend similar to that exhibited in the plots of the binding energies, the enhancement being much greater for the polar quantum dots. In the bulk limit, with the PB potential, we see that the oscillator strength is actually almost 7 times (5 times) larger than the value obtained with the Coulomb potential for $\mathrm{CuCl}$ $(\mathrm{ZnO})$. Although both $f_{\mathrm{ex}}$ and $f_{\mathrm{ex}}^{(s)}$ increase with the decreasing dot size, their ratio starts to get smaller and approaches unity in the high confinement limit. This is because in very small dots, the confinement effect takes over and strongly suppresses the correlation between the electron and the hole, thereby reducing the significance of the Coulomb interaction.

We have used an effective mass approximation to calculate the energy levels of electrons and holes and the binding energies of excitons in ionic quantum dots. This approximation becomes less valid for the electron and the hole energy levels when the dot sizes are smaller than the corresponding Bohr radii. However, as we are primarily interested in investigating the changes in the exciton binding energies and oscillator strengths due to the exciton-phonon interaction, we believe our results are fairly meaningful even in smaller dots.

In summary, we have calculated the variations of the binding energy and the oscillator strength of the ground state of an exciton in an ionic quantum dot as a function of the dot size using a parabolic confining potential. The strong exciton-phonon interaction is taken into account by using an effective potential between the electron and the hole as derived by Pollmann and Büttner. The values of the exciton binding energies thus calculated are compared with those obtained using statically screened Coloumb potential and are found to be considerably larger for all values of the quantum dot size. The confinement effects enhance the effective interaction between an electron and a hole and thus increase the exciton binding

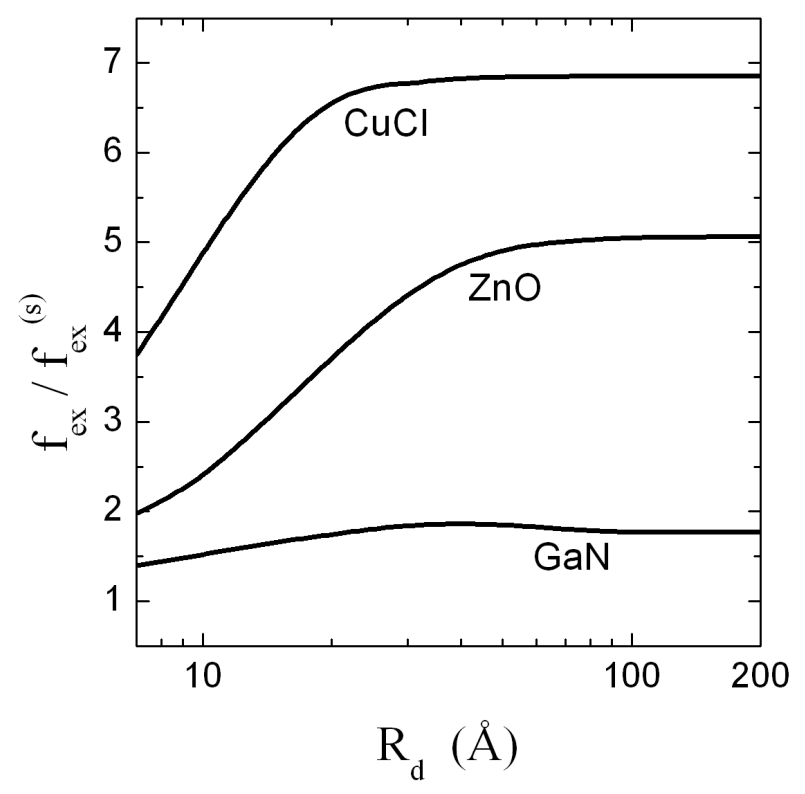

Fig. 3 Variation of the ratio of oscillator strengths as a function of confinement in quantum dots of $\mathrm{GaN}, \mathrm{ZnO}$ and $\mathrm{CuCl}$. $f_{\mathrm{ex}}$ and $f_{\mathrm{ex}}^{(s)}$ are the oscillator strengths as calculated using PollmannBüttner and statically screened Coulomb potentials, respectively. 
energies. However, the enhancement of the effective interaction does not lead to its dominance in small quantum dots as the kinetic energies and the single particle energies scale at a much faster rate than the interaction energy so that the electron-hole correlation is reduced. A signature of such a trend is seen in the behavior of the oscillator strength, where it is seen that with the increase of the degree of confinement the oscillator strength of the exciton ground state becomes less dependant on the form of the effective interaction.

\section{References}

[1] A. D. Yoffe, Adv. Phys. 50, 1 (2001).

[2] P. Michler, A. Kiraz, C. Becher, W. V. Schönfeld, P. M. Petroff, L. Zhang, E. Hu, and A. İmamoğlu, Science 290, 2282 (2000).

[3] Y. Arakawa and H. Sakaki, Appl. Phys. Lett. 40, 939 (1982).

[4] P. Ramvall, P. Riblet, S. Nomura, Y. Aoyagi and S. Tanaka, J. Appl. Phys. 87, 3883 (2000).

[5] T. Tawara, S. Tanaka, H. Kumano, and I. Suemune, Appl. Phys. Lett. 75, 235 (1999).

[6] Z. K. Tang, G. K. L. Wong, P. Yu, M. Kawasaki, A. Ohtomo,, H. Koinuma, and Y. Segawa, Appl. Phys. Lett. 72, 3270 (1998).

[7] M. Lu, X. J. Yang, S. S. Perry, and J. W. Rabalais, Appl. Phys. Lett. 80, 2096 (2002).

[8] S. Yano, T. Goto, and T. Itoh, J. Appl. Phys. 79, 8216 (1996).

[9] P. Nandakumar, C. Vijayan, and Y. V. G. S. Murti, J. Appl. Phys. 91, 1509 (2002).

[10] P. Ramvall, P. Riblet, S. Nomura, Y. Aoyagi, and S. Tanaka, J. Appl. Phys 87, 3883 (2000).

[11] S. Nomura and T. Kobayashi, Phys. Rev. B 45, 1305 (1992).

[12] S. Schmitt-Rink, D. A. B. Miller, and D. S. Chemla, Phys. Rev. B 35, 8113 (1987).

[13] M. C. Klein, F. Hache, D. Ricard, and C. Flytzanis, Phys. Rev. B 42, 11123 (1990).

[14] J. C. Marini, B. Strebe, and E. Kartheuser, Phys. Rev. B 50, 14302 (1994).

[15] K. Oshiro, K. Akai, and M. Matsuura, Phys. Rev. B 59, 10850 (1999).

[16] H. Ajiki, phys. stat. sol. (b) 224, 633 (2001).

[17] H. Haken, Z. Phys. 146, 527 (1956).

[18] J. Pollmann and H. Büttner, Solid State Commun. 17, 1171 (1975).

[19] J. Pollmann and H. Büttner, Phys. Rev. B 16, 4480 (1977).

[20] S. Bednarek, J. Adamowski, and M. Suffczynski, Solid State Commun. 21, 1 (1977).

[21] E. O. Kane, Phys. Rev. B 18, 684 (1978).

[22] L. F. Register, Phys. Rev. B 45, 8756 (1992).

[23] S. M. Komirenko, K. W. Kim, M. A. Stroscio, and M. Dutta, Phys. Rev. B 59, 5013 (1999).

[24] H. Rücker, E. Molinari, and P. Lugli, Phys. Rev. B 44, 3463 (1991).

[25] E. Molinari, C. Bungaro, M. Gulia, P. Lugli, and H. Rücker, Semicond. Sci. Technol. 7, B67 (1992).

[26] R. Jalabert and S. Das Sarma, Phys. Rev. B 40, 9723 (1989).

[27] G. Behnke, H. Büttner, and J. Pollmann, Solid State Commun. 20, 873 (1976).

[28] C. H. Henry and K. Nassau, Phys. Rev. B 1, 1628 (1970).

[29] G. W. Bryant, Phys. Rev. B 37, 8763 (1988).

[30] W. Que, Phys. Rev. B 45, 11036 (1992).

[31] Landolt-Börnstein, Numerical Data and Functional Relationships in Science and Technology, New Series, Group III, Vol. 17, Sub Volume b, edited by O. Madelung (Springer, New York, 1982).

[32] V. Bougrov, M. E. Levinshtein, S. L. Rumyantsev, and A. Zubrilov, in Properties of Advanced Semiconductor Materials GaN, AlN, InN, BN, SiC, SiGe, edited by M. E. Levinshtein, S. L. Rumyantsev, M. S. Shur (John Wiley and Sons, Inc., New York, 2001), pp. 1-30.

[33] K. K. Bajaj, phys. stat. sol. (b) 64, K107 (1974). 